\title{
A STUDY OF SOLVING SYSTEM OF LINEAR EQUATION USING DIFFERENT METHODS AND ITS REAL LIFE APPLICATIONS:
}

\author{
Khushbu kumari ${ }^{\mathrm{a}} \mathrm{R}$ K Poonia ${ }^{\mathrm{b}}$ \\ khudhbookumari06294@gmail.comªrkpooniae7260@cumail.in ${ }^{b}$ \\ DEPARTMENT OF MATHEMATICS \\ CHANDIGARH UNIVERSITY, GHARAUN, MOHALI, PUNJAB
}

\begin{abstract}
Solving system of linear equation (or linear systems or, also simultaneous equation) is a common situation in many scientific and technological problems. Many method either analytical or numerical, have been developed to solve them so, in this paper I will explain how to solve any arbitrary field using the different - different methods of system of linear equation for this we need to define some concepts. Like a general method most used in linear algebra is the Gauss Elimination or variation of this sometimes they are referred as "direct methods "Basically it is an algorithm that transforms the system into an equivalent one but with a triangular matrix, thus allowing a simpler resolution,

Other methods can be more effective in solving system of linear equation like Gauss Elimination or Row Reduction, Gauss Jordan and Crammer's rule etc.So, in this paper I will explain these method by taking an example also, in this paper I will explain the Researcher's works that how they explain different -different methods by taking different example. And I worked on using these different methods in solving a single example, i.e. I will use these methods in an example. In this paper I will explain the real life application that how System of Linear Equation used in our daily life.
\end{abstract}

KEYWORDS:System of linear equation, Gauss elimination methods (or Row Reduction), Gauss Jordan methods, Crammers rule, and applications.

\section{INTRODUCTION:}

A system of equation is a set or collection of equation solved together. Collection of linear equation is termed as system of linear equations. They are often based on same set of variables. Or a linear system of equation is a system where the output is proportionate to the input.

For example: If the business organization is a linear system, then if we double the capital, employees, raw materials and factories ( the inputs) then we expect to get double the production (the outputs).

Various methods have been evolved to solve the linear equations. Various methods are proposed by different mathematiciansDifferent methods are being used for the solution of system of linear equation. This paper deals with the Gaussian Elimination methods, Gauss Jordan methods, crammers rule and some other rules or methods. Not a single method which is used to be best method to get appropriate result. Speed used as a significant factor for the solution of system of linear equations as the volume of computation engaged are to large. The solution of a linear system of equation can be accomplished by a numerical method which directs method. Amongst 
the direct method, they will describe the elimination method by gauss as also its modification and the LU decomposition method. Numerical analysis, the subject which studies algorithms for computing expressions defined with real numbers. Such systems can involve many equations in many variables. So after reviewing methods for solving two linear equations in two variables, we use matrices and matrix operations to develop procedures that are suitable for solving linear systems of any size.

\section{HISTORY:}

System of equation arose in Europe with the introduction in 1637 by Rene Descartes. System of linear equation is given by different-different mathematicians. Linear equation were invented in 1843 by Irish mathematicians Sir William Rowan Hamilton.

\section{RELATIVE WORKS:}

When it comes to systems of linear equations, there is not much research pertaining to what methods students tend to use, especially when multiple choice answers are involved. There is research on linear equations and research on multiple choice answers, but combining the two together and going one step further to make linear equations into systems of linear equations has not been well established (Anderson, 1989; Coppedge\& Hanna, 1971; Hewitt, 2012; Huntley, Marcus, Kahan, \& Miller, 2007; Kazemi, 2002; Marshall, 1983; Nogueira de Lima \& Tall, 2008).

Over the years, many methods have been devised to solve system of linear equations for two variables and more than two variables.

Cornelius Lanczos [1952] A simple algorithm is described which is well adapted to the effective solution of large systems of linear algebraic equations by a succession of well-convergent approximations

FALL [2012]. This descriptive study focuses on the approaches college students (ages 20 - 24) use when solving systems of linear equations problems that have multiple choice answers. Participants were from a midsize public university in the northeast. Four approaches were considered - three forwards approaches: 1) substitution, 2) elimination, and 3) graphing, and one backwards approach: plugging in the $\mathrm{x}$ and $\mathrm{y}$ values from each multiple choice option. Participants solved systems of linear equations problems and answered questions based on their methods in a structured clinical interview

KHAN et al. [2015]. This paper comprises of matrix introduction, and the direct methods for linear equations. The goal of this research was to analyze different elimination techniques of linear equations and measure the performance of Guassian elimination and Guass Jordan method, in order to find their relative importance and advantage in the field of symbolic and numeric computation. The purpose of this research is to revise an introductory concept of linear equations, matrix theory and forms of Guassian elimination through which the performance of Guass Jordan and Guassian elimination can be measured.

Riga [2016].The systems of linear equations are a classic section of numerical methods which was already known BC. It reached its highest peak around 1600-1700 due to the public demand 
for solutions of technical and engineering tasks, nevertheless, it is still topical nowadays. This paper describes another iterative approach to solving linear systems, which is based in the multiple transfers of the solution proximity point towards the solution itself, simultaneously reducing the differences of all the system equations

MARYA et.al [2017]. In this paper we present a survey of three direct methods for the solution of system of linear equations. Various methods are introduced to solve systems of linear equations but in contrast to all methods not a single method are best for all situations (to get appropriate solution). These methods depend on speed and accuracy as these are an important factor in solving large systems of equations because the volume of computations involved for solution are bulky. Direct solution of simultaneous linear equations may be regarded as a slow process for large systems of equations and requires special treatment to avoid numerical instability.

https://www.khanacademy.org/commoncore/grade-HSA-A-REI\#HSA-REI.C.6The elimination method is a technique for solving systems of linear equations. This article reviews the technique with examples and even gives you a chance to try the method yourself. ICTE 2016,

.Maharaja [2018]. This paper focused on the written work of two students to questions based on the solution of a system of linear equations using matrix methods. The objective was to gauge the possible level of mathematical understanding of the students by using a framework that was arrived at. That framework was used to analyse the level of mathematical understanding of those students' written responses to the questions, with the focus on their use of symbolic language. It was found that the framework enabled the researcher to get a deeper insight into those students use of the symbolic language, used both in an instrumental role and also as a communicative function.

Then Sahgal [2018], Mafteiu-scai.[2012],Flag.[2017], Tofigh [2004],Allahviranloo.[2005], and many more.

\section{DEFINITION:}

A system of linear equations is a collection of one or more linear equations involving the same set of variables. Or Linear systems are systems of equations in which the variables are never multiplied with each other but only the constants and then summed up.

A linear equation in variables $a_{1} x_{1}+a_{2} x_{2}+a_{3} x_{3}+\cdots . . a_{n} x_{n}=\mathrm{b}$

Where $a_{1}, a_{2}, a_{3} \ldots \ldots \ldots \ldots a_{n}$ and $\mathrm{b}$ are constants of real and complex numbers. The constant $a_{i}$ is called the coefficient of $x_{i}$ and $\mathrm{b}$ is called the constant term of the equation.

\section{METHODS THAT ARE USED TO SOLVE SYSTEM OF LINEAR EQUATIONS:}

There are so many methods are used to solve the linear system of equation. Some of these are:elimination of variables, Row reduction(Gauss elimination method), Cramer's rule,and etc.. 
5.1 ELIMINATION OF VARIABLES: The simplest method for solving a system of linear equations is to repeatedly eliminate variables. This method can be described as follows:

1. In the first equation, solve for one of the variables in terms of the others.

2. Substitute this expression into the remaining equations. This yield a system of equations with one fewer unknown.

3. Repeat until the system is reduced to a single linear equation.

Solve this equation, and then back - substitute until entire solution is found

\subsection{Row Reduction Method:}

To perform row reduction in a matrix, one uses a sequence of elementary row operations to modify the matrix until the lower half hand corner of the matrix is filled with zeros, as much as possible.

For example:- solve the system of equation using Row reduction method

$$
\begin{aligned}
& x+4 y+9 z=16 \\
& 2 x+y+z=10 \\
& 3 x+2 y+3 z=18
\end{aligned}
$$

write it as $\mathrm{AX}=\mathrm{B}$

$$
\left[\begin{array}{lll}
1 & 4 & 9 \\
2 & 1 & 1 \\
3 & 2 & 3
\end{array}\right] \quad\left(\begin{array}{l}
x \\
y \\
z
\end{array}\right)=\left(\begin{array}{l}
16 \\
10 \\
18
\end{array}\right)
$$

Augmented matrix ; $[A: B]$

$$
\left[\begin{array}{llll}
1 & 4 & 9 & : 16 \\
2 & 1 & 1 & : 10 \\
3 & 2 & 3 & : 18
\end{array}\right]
$$

apply $R_{2} \rightarrow R_{2}-2 R_{1} \quad, R_{3} \rightarrow R_{3}-3 R_{1}$

apply $R_{2} \rightarrow R_{2}-2 R_{1} \quad, R_{3} \rightarrow R_{3}-3 R_{1}$

$$
\begin{aligned}
& {\left[\begin{array}{cccl}
1 & 4 & 9 & : 16 \\
0 & -7 & -17 & :-32 \\
0 & -10 & -24 & :-30
\end{array}\right]} \\
& R_{3} \rightarrow 7 R_{3}-10 R_{2} \\
& {\left[\begin{array}{cccl}
1 & 4 & 16 & : 16 \\
0 & -7 & -17 & :-22 \\
0 & 0 & 2 & : 10
\end{array}\right]}
\end{aligned}
$$

$x+4 y+9 z=16$ 


$$
\begin{aligned}
-7 y-17 z & =-22 \\
2 z & =10
\end{aligned}
$$

$\mathrm{Z}=5, \mathrm{y}=-9$ and $\mathrm{x}=7$

\subsection{Same question solved by guass Jordan method:}

$$
\begin{array}{r}
x+4 y+9 z=16 \\
2 x+y+z=10 \\
3 x+2 y+3 z=18
\end{array}
$$

write it as $\mathrm{AX}=\mathrm{B}$

$$
\left[\begin{array}{lll}
1 & 4 & 9 \\
2 & 1 & 1 \\
3 & 2 & 3
\end{array}\right] \quad\left(\begin{array}{l}
x \\
y \\
z
\end{array}\right)=\left(\begin{array}{l}
16 \\
10 \\
18
\end{array}\right)
$$

Augmented matrix ; $[A: B]$

$$
\begin{aligned}
& {\left[\begin{array}{llll}
1 & 4 & 9 & : 16 \\
2 & 1 & 1 & : 10 \\
3 & 2 & 3 & : 18
\end{array}\right]} \\
& \text { Apply } R_{2} \rightarrow R_{2}-2 R_{1} \quad, R_{3} \rightarrow R_{3}-3 R_{1} \\
& \text { Apply } R_{2} \rightarrow R_{2}-2 R_{1} \quad, R_{3} \rightarrow R_{3}-3 R_{1} \\
& {\left[\begin{array}{cccl}
1 & 4 & 9 & : 16 \\
0 & -7 & -17 & :-32 \\
0 & -10 & -24 & :-30
\end{array}\right]} \\
& R_{3} \rightarrow 7 R_{3}-10 R_{2} \\
& {\left[\begin{array}{cccl}
1 & 4 & 16 & : 16 \\
0 & -7 & -17 & :-22 \\
0 & 0 & 2 & : 10
\end{array}\right] R_{3} \rightarrow 7 R_{3}-10 R_{2}, R_{1} \rightarrow 7 R_{1}+4 R_{2}} \\
& {\left[\begin{array}{cccc}
7 & 0 & -5 & : 24 \\
0 & -7 & -17 & :-22 \\
0 & 0 & 2 & : 10
\end{array}\right] R_{1} \rightarrow 2 R_{1}+5 R_{3} \quad R_{2} \rightarrow 2 R_{2}+17 R_{3}} \\
& {\left[\begin{array}{cccc}
14 & 0 & 0 & : 98 \\
0 & -14 & 0 & : 126 \\
0 & 0 & 2 & : 10
\end{array}\right]} \\
& 14 \mathrm{x}=98
\end{aligned}
$$


$-14 y=126$

$2 \mathrm{z}=10$

So, $\mathrm{x}=7, \mathrm{y}=-9$, and $\mathrm{z}=5$

5.5 Same question solved by crammers rule :

$$
\begin{aligned}
& x+4 y+9 z=16 \\
& 2 x+y+z=10 \\
& 3 x+2 y+3 z=18
\end{aligned}
$$

First we need to find the value of delta $\Delta$

$$
\Delta=\left|\begin{array}{lll}
1 & 4 & 9 \\
2 & 1 & 1 \\
3 & 2 & 3
\end{array}\right|
$$

Now we have to apply $3^{\text {rd }}$ row determinant rule

$1\left|\begin{array}{ll}1 & 1 \\ 2 & 3\end{array}\right|-4\left|\begin{array}{ll}2 & 1 \\ 3 & 3\end{array}\right|+9\left|\begin{array}{ll}2 & 1 \\ 3 & 2\end{array}\right|$

$(3-2)-4(6-3)+9(2-3)$

$1-12+9$

$10-12=-2 \quad$ so $\Delta=-2$

Now for finding the value of $\mathrm{X}, \mathrm{Y}$ and $\mathrm{Z}$ we need to find the value of $\Delta x, \Delta y$ and $\Delta z$ In $\Delta 1^{\text {st }}$ column we have to replace by these three exponent

$$
\begin{aligned}
& \Delta x=\left|\begin{array}{lll}
16 & 4 & 9 \\
10 & 1 & 1 \\
18 & 2 & 3
\end{array}\right| \\
& 16(3-2)-4(30-18)+9(20-18) \\
& 16-48+18=-14
\end{aligned}
$$

So $\quad x=\frac{\Delta x}{\Delta} \frac{-14}{-2}=7$ i.e $x=7$ 


$$
\begin{aligned}
& \Delta y=\left|\begin{array}{lll}
1 & 16 & 9 \\
2 & 10 & 1 \\
3 & 18 & 3
\end{array}\right| \\
& 1(30-18)-16(6-3)+9(36-30) \\
& 12-48+54=18 \\
& y=\frac{\Delta y}{\Delta}=\frac{18}{-2}=-9 \\
& \Delta z=\quad \begin{array}{lll}
1 & 4 & 16 \\
3 & 1 & 10 \\
3 & 2 & 18
\end{array} \mid \\
& 1(18-20)-4(36-30)+16(4-3) \\
& -2-24+16=-10 \\
& z=\frac{\Delta z}{\Delta}=\frac{-10}{-2}=5
\end{aligned}
$$

So, the same question solved by these methods we get the same values of $\mathrm{x}, \mathrm{y}$ and $\mathrm{z}$

Similarly we can solve these type of question by using different -different methods of system of linear equation very easily

\section{REAL LIFE APPLICATIONS OF SYSTEM OF LINEAR EQUATION:}

There are so many applications of system of linear equation in which some of them are explained by using the example :

There are two cars moving towards each other and are 500 miles apart. One car is moving at a speed of $100 \mathrm{mph}$ and the other is moving at $70 \mathrm{mph}$. Assuming that the cars start moving at the same time how long does it take for the two cars to meet? Here let $t$ represent the amount of time that the cars are traveling before they meet. Now, we need to sketch a figure for this one. This figure will help us to write down the equation that we'll need to solve 


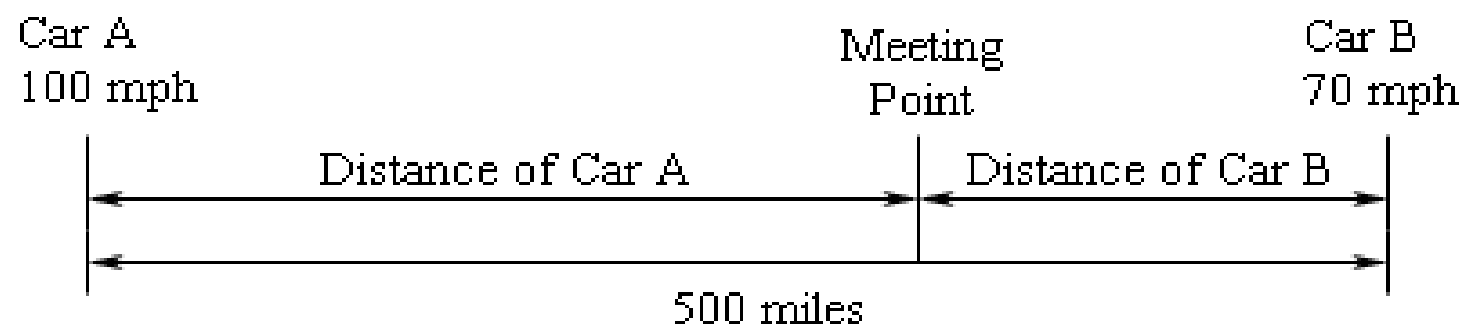

From this figure, we can see that the Distance Car A travels plus the Distance Car B travels must equal the total distance separating the two cars, 500 miles Here is the linear equation in two separate forms.

The distance of Car A + Distance of Car B $=500$

Rate of Car A * Time of Car A + Rate of Car B * Time of Car B $=500$

We used the standard formula here twice, once for each car. We know that the distance a car travels is the rate of the car times the time traveled by car. In this case, we know that Car A travels at $100 \mathrm{mph}$ for $\mathrm{t}$ hours and that

Car B travels at $80 \mathrm{mph}$ for thours as well. Plugging these into the linear equation and solving it gives us,

$100 \mathrm{t}+70 \mathrm{t}=500$

$170 \mathrm{t}=500$

$t=500 / 170$

$=2.941176 \mathrm{hrs}$

So, they will travel for approximately 2.94 hours to meet

\section{Health Care Professional:}

The health care field, including doctors and nurses, often use linear equations to calculate medical doses. Linear equations are also used to determine how different medications may interact with each other and how to determine correct dosage amounts to prevent overdose with patients using multiple medications. Doctors also use linear equations to calculate doses based on a patient's weight.

\section{Financial Analyst:}

Financial occupations often require the use of linear equations. Accountants, auditors, budget analysts, insurance underwriters and loan officers frequently use linear equations to balance accounts, determine pricing and set budgets. Linear equations used in financial occupations may also be used in creating family budgets as well. A financial planner, for example, uses linear equations to determine the total worth of a client's stocks. 


\section{Architect and Builder :}

The construction field frequently uses linear equations when measuring and cutting all types of materials for job sites. Both carpenters and electricians are included in the construction field and use linear equations on many of the jobs they do. A carpenter might, for example, use a linear equation to estimate the cost of wood and nails for a remodeling project.

\section{Computer Programmer :}

Computer programmers and support specialists must be able to solve linear equations. Linear equations are used within software applications, on websites and security settings, which must be programmed by a computer programmer. Support specialists must be able to understand linear equations to troubleshoot many software and networking issues. A programmer, for example,might use linear equations to calculate the time needed to update a large database of information

\section{CONCLUSION:}

Based on the readings and observations and articles, we have concluded from this paper that we can solve any arbitrary question and two variables and more than two variable question can be easily solved by using methods of system of linear equation. Also as I explained in this paper that same question can be solved by more than two methods so, I also concluded, a question is not particularly solved by only a specific method we can also solved that by using different different methods as I have proven in this paper. Also we can use system of linear equation in daily life .

Direct methods are not appropriate for solving large number of equations in a system, particularly when the coefficient matrix is sparse, i.e. when most of the elements in a matrix are zero as contrast to LU decomposition method

\section{References:}

1. Noreen Jamil,“ direct and indirect solvers for linear system equations”, june 2012.

2.Froilán M. Dopico, “Alan Turing and the origins of modern Gaussian elimination”, Institutode CienciasMatemáticas CSIC-UAM-UC3M-UCM, December 14, 2012.

3. Yadanar Mon, Lai lai win kyi, "performance comparison of guassian elimination and guass Jordan”, International Journal of Computer \& Communication Engineering Research (IJCCER), Information Technology Department, Volume 2,02- march-2014.

4. Luke Smith and Joan Powell: “An Alternative Method to Gauss-Jordan Elimination: Minimizin g Fraction Arithmetic”, The Mathematics Educator, 2011

5. J. F. Grcar, "How ordinary elimination became Gaussian elimination”. Historia Math., 38(2):163_218, 2011.

6. Etienne Loute, “guassian elimination as computational paradigm”, August 2003. 
7. NaeKaunTsao, Ditroit Michigan, "On the Equivalence of Gaussian Eliminatio and GaussJordan Reduction in Solving Linear Equations”, February 1989.

8.Yadav, A. S. and Kumar Ashish, "Numerical Solution of System of Linear Equations by Iterative methods”, International Journal of Engineering Sciences \& Research Technology, Vol.

9. Issue 1, pp. 203-208, 2017. Available:

10.Sastry, S. S., "Introductory Methods of Numerical Analysis", Book of Numerical analysis, 5th edition.

11. Noreen, J. "A Comparison of Direct and Iterative Solvers for Linear System of Equations”, International Journal Emerging Science, Vol. 2, Issue 2, pp. 310- 321, 2012. [4] Dass H. K. and Verma R., "Numerical solution of linear equation solve by Direct and Iterative methods”, Book Numerical analysis, Vol. 3, pp. 566-580, 2010.

12. Dutta, B. N., "Numerical Linear Algebra and Applications” Brookes/Cole publishing company, 1995. [6] Wen Li, "Comparison results for solving preconditioned linear systems”. Journal of and Applied Mathematics journal 182: pp 319-329, 2005

13. Lay DC 2006. Linear Algebra and its Applications. $3{ }^{\text {rd }}$ Edition. New York: Pearson.

14.Lazebnik F 1996. On Systems of Linear Diophantine.Equations. From $<$ http://www.math.udel.edu/ lazebnik/

15.papers/dioph2.pdf $>$ (Retrieved on 20 February 2015). Maharaj A 2010. An APOS analysis of students' under-

16.standing of the concept of a limit of a function.Pythagoras, 71: 4152.

17.Maharaj A 2013. An APOS analysis of natural sciencestudents' understanding of derivatives. South Afri-can Journal of Education, 33(1): 146-164.

18. Maharaj A 2014. An APOS analysis of natural science stu-dents' understanding of integration. REDIMAT - Journal

19. of Research in Mathematics Education, 3(1): 53-72.Maharaj A 2015. A framework to gauge mathematicalunderstanding: A case study on linear algebra con-

cepts. International Journal of Educational Scienc-es, 11(2): 144-153.

20. Ndlovu ZA, Brijlall D 2015. Pre-service teachers' men-tal constructions of concepts in matrix algebra: ASouth African case. African Journal of Research inMathematics, Science and Technology Education,19(2): 156-171. 
21.Nickerson RS 1985. Understanding understanding. American Journal of Education, 93(2): 201-239.Sierpinska A 1994. Understanding in Mathematics.London: The Falmer Press.

22. Sierpinska A, Nnadozie A, Okta A 2002. A Study ofRelationships between Theoretical Thinking andHigh Achievement in Linear Algebra. Manuscript.Montreal, Quebec: Concordia University.

23. Skemp $\mathrm{R}$ 1976. Relational understanding and instrumen-tal understanding. Mathematics Teaching, 77: 20-26.Stewart S, Thomas M 2009. Linear Algebra Snapshotsthrough APOS and Embodied, Symbolic and FormalWorlds of Mathematical Thinking. From $<$ http://www.merga.net.au/documents/Stewart_RP09.pdf $>$ (Retrieved on 11 November 2013).

24. Stylianides AJ, Stylianides GJ 2007. Learning Mathe-matics with Understanding: A Critical Considerationof the Learning Principle in the Principles and Stan-dards for School Mathematics.From <http://www.math.umt.edu/tmme/vol4no1/TMMEv4n1a8.pdf > (Retrieved on 6 August 2013).

25. vanced Mathematical Thinking. Dordrecht, Nether-lands: Kluwer Academic Publishers, pp. 65-81.Zaslavsky O, Shir K 2005. Students' conceptions of a mathematical definition. Journal for Research inMathematics Education, 36(4): 317-346. Weller K, Arnon I, Dubinsky E 2009. Pre-service teach-ers' understanding of the relation between a fractionor integer and its decimal expansion. Canadian Jour-nal of Science, Mathematics and Technology Edu-cation, 9(1): 5-28.

26. Paper received for publication on October 2015Paper accepted for publication on December 20. 